\title{
Use of the oral beta blocker bisoprolol to reduce the rate of exacerbation in people with chronic obstructive pulmonary disease (COPD): a randomised controlled trial. (BICS)
}

\section{Seonaidh Cotton ( $\square$ s.c.cotton@abdn.ac.uk)}

University of Aberdeen https://orcid.org/0000-0002-7883-0608

Graham Devereux

Liverpool School of Tropical Medicine https://orcid.org/0000-0002-0024-4887

Hassan Abbas

University of Aberdeen

Andrew Briggs

University of Glasgow

Karen Campbell

University of Aberdeen

Rekha Chaudhuri

University of Glasgow

Gourab Choudhury

NHS Lothian

Dana Dawson

University of Aberdeen

Anthony De Soyza

University of Newcastle

Shona Fielding

University of Aberdeen

Simon Gompertz

Queen Elizabeth Hospital Birmingham

John Haughney

University of Aberdeen

Chim Lang

University of Dundee

Amanda Lee

University of Aberdeen

Graeme MacLennan

University of Aberdeen 


\section{William MacNee}

The University of Edinburgh

\section{Kirsty McCormack}

University of Aberdeen

\section{Nicola McMeekin}

University of Glasgow Institute of Health and Wellbeing

\section{Nicholas Mills}

The University of Edinburgh

\section{Alyn Morice}

University of Hull

John Norrie

The University of Edinburgh

\section{Mark Petrie}

University of Glasgow

\section{David Price}

University of Aberdeen

\section{Philip Short}

NHS Tayside

\section{Jorgen Vestbo}

The University of Manchester

\section{Paul Walker}

Liverpool University Hospitals NHS Foundation Trust

\section{Jadwiga Wedzicha}

Imperial College London National Heart and Lung Institute

\section{Andrew Wilson}

University of East Anglia

\section{Brian Lipworth}

University of Dundee

\section{Research Article}

Keywords: COPD, exacerbation, randomised controlled trial, bisoprolol, beta blocker

Posted Date: February 9th, 2022

DOI: https://doi.org/10.21203/rs.3.rs-1271797/v1

License: (c) (1) This work is licensed under a Creative Commons Attribution 4.0 International License.

Read Full License 
Version of Record: A version of this preprint was published at Trials on April 14th, 2022. See the published version at https://doi.org/10.1186/s13063-022-06226-8. 


\section{Abstract}

Background: Chronic Obstructive Pulmonary Disease (COPD) is associated with significant morbidity, mortality and healthcare costs. Beta-blockers are well-established drugs widely used to treat cardiovascular conditions. Observational studies consistently report that beta-blocker use in people with COPD is associated with a reduced risk of COPD exacerbations. The bisoprolol in COPD study (BICS) investigates whether adding bisoprolol to routine COPD treatment has clinical and cost-effective benefits. A sub-study will risk stratify participants for heart failure to investigate whether any beneficial effect of bisoprolol is restricted to those with unrecognised heart disease.

Methods: BICS is a pragmatic randomised parallel group double-blind placebo-controlled trial conducted in UK primary and secondary care sites. The major inclusion criteria are an established predominant respiratory diagnosis of COPD (post-bronchodilator $\mathrm{FEV}_{1}<80 \%$ predicted, $\mathrm{FEV}_{1} / \mathrm{FVC}<0.7$ ), a self-reported history of $\geq 2$ exacerbations requiring treatment with antibiotics and/or oral corticosteroids in a 12-month period since March 2019, age $\geq 40$ years and a smoking history $\geq 10$ pack years. A computerised randomisation system will allocate 1574 participants with equal probability to intervention or control groups, stratified by centre, and recruitment in primary/secondary care. The intervention is bisoprolol (1.25 mg tablets) or identical placebo. The dose of bisoprolol/placebo is titrated up to a maximum of 4 tablets a day (5mg bisoprolol) over four-seven weeks depending on tolerance to up-dosing of bisoprolol/placebo - these titration assessments are completed by telephone or video call. Participants complete the remainder of the 52-week treatment period on the final titrated dose (1,2,3,4 tablets) and during that time are followed up at 26 and 52 weeks by telephone or video call. The primary outcome is the total number of participant reported COPD exacerbations requiring oral corticosteroids and/or antibiotics during the 52-week treatment period. A sub-study will risk stratify participants for heart failure by echocardiography and measurement of blood biomarkers.

Discussion: The demonstration that bisoprolol reduces the incidence of exacerbations would be relevant not only to patients and clinicians but also to healthcare providers, in the UK and globally.

Trial registration: Current controlled trials ISRCTN10497306 (registered 16 August 2018)

\section{Introduction}

\section{[6a] Background/Rationale}

Chronic obstructive pulmonary disease (COPD) is a lung disease characterised by progressive airflow obstruction. ${ }^{1}$ Globally 210 million people have moderate to severe COPD and prevalence is increasing. ${ }^{2,3}$ COPD is the fifth leading cause of death globally and by 2030 it is expected to be the fourth, accounting for $8 \%$ of deaths. ${ }^{4}$ In 2002, COPD was the eleventh leading cause of disability adjusted life years (DALY)s, by 2030 it is expected to be the seventh. ${ }^{4}$ In the UK, the prevalence of diagnosed COPD has increased from about 991,000 in 2004 to 1.2 million in $2012,{ }^{5}$ it is the fifth leading cause of death, accounting for 
about 30,000 deaths annually. The progressive airflow limitation of COPD is associated with increasing disability, work absence, long-term morbidity, physical and psychological co-morbidities and premature mortality. People with COPD are more likely to have associated comorbidities, including ischaemic heart disease, hypertension, diabetes, and depression, and unrecognised heart failure has been reported in up to $20 \%$ of COPD patients. ${ }^{6-12}$

Acute deteriorations in symptoms known as exacerbations are an important clinical feature of COPD. They are associated with accelerated lung function decline, reduced physical activity, reduced quality of life and increased mortality. ${ }^{13-16}$ COPD is one of the costliest inpatient conditions and exacerbations account for about $60 \%$ of the $£ 1$ billion NHS expenditure on COPD. ${ }^{1,17}$ Despite advances in management there is still an unmet need for improved pharmacological treatment of COPD particularly the prevention of exacerbations.

Beta-blockers are a class of drug with proven benefit in people with heart failure or ischemic heart disease, particularly those with left ventricular impairment. The rationale for repurposing beta-blockers for use in COPD comes from the findings of observational cohort studies that beta-blocker use in people with COPD is associated with a reduced risk of exacerbation. ${ }^{18-24} \mathrm{~A}$ systematic review of 15 studies of betablocker use for cardiovascular disease demonstrated that beta-blocker use in COPD patients was associated with a $28 \%(95 \% \mathrm{Cl} 17-37)$ reduction in mortality, and a $37 \%(95 \% \mathrm{Cl} 29-43)$ reduction in exacerbations. ${ }^{18}$ The mechanisms by which beta-blockers may reduce exacerbations remains uncertain although it is biologically plausible that some of the episodes conventionally diagnosed as acute exacerbations of COPD are in reality cardiac events for which beta-blockers have proven benefits. ${ }^{25,26}$

Beta-blockers competitively antagonise the effects of catecholamines on beta-adrenoreceptors. Beta1adrenoreceptors are found only in the heart, whereas beta2-adrenoreceptors are more ubiquitous, being found in the heart and lungs. Bronchodilating beta2-agonists are the mainstay of COPD and asthma treatment and beta-blockers that antagonise beta2-agonists could have adverse respiratory effects, and for asthma, a condition with reversible airflow limitation, beta-blockers are usually avoided. ${ }^{27} \mathrm{~A}$ systematic review of randomised controlled trials (RCTs) studying the effects of beta1-selective blockers in people with COPD reported that repeat administration of beta1-selective blockers was not associated with an increase in respiratory symptoms, change in lung function or a reduction in response to inhaled beta2-agonist. ${ }^{28}$ Clinical evidence is supportive of beta1-blockers such as bisoprolol being safe in COPD and beta1-selective blocker use in COPD patients with heart failure is a guideline recommendation. ${ }^{29,30}$

We describe here the bisoprolol in COPD study (BICS) which tests the hypothesis that adding bisoprolol to routine COPD treatment reduces the rate of exacerbation. A sub-study will test the hypothesis that any beneficial effect of bisoprolol is restricted to those with unrecognised heart disease. The full protocol is available as a supplemental file.

\section{[7] Objectives}


The primary objective is to determine the clinical and cost-effectiveness of adding bisoprolol to usual COPD treatment in patients with COPD at high risk of exacerbation as evidenced by a history of at least two COPD exacerbations in a previous year.

The secondary objectives are to compare the following outcomes between participants treated with bisoprolol, and those treated with placebo:

a. Hospital admissions with a primary diagnosis of COPD exacerbation.

b. Time to first exacerbation of COPD.

c. Total number of emergency hospital admissions.

d. Total number of major adverse cardiovascular events (MACE).

e. Lung function.

f. Changes in breathlessness during treatment.

g. All-cause, respiratory and cardiac mortality.

h. Drug reactions and serious adverse events.

i. Health related quality of life.

j. Disease specific health status.

k. Health care utilisation.

I. Incremental cost-per-exacerbation avoided.

m. Costs to the NHS and patients and lifetime cost-effectiveness based on extrapolation modelling.

n. Modelled lifetime incremental cost per Quality Adjusted Life Year.

The sub-study will risk stratify participants to investigate

o. Treatment effects in participants with and without unrecognised heart disease.

\section{[8] Trial design}

BICS is a pragmatic randomised, double-blind, parallel group, placebo-controlled, multicentre clinical trial investigating whether bisoprolol is superior to placebo when added to current COPD therapy for 52 weeks in patients with COPD who have had two or more exacerbations of COPD in a previous year treated with oral corticosteroids (OCS) and/or antibiotics. Figure 1 provides a schematic representation of study design and schedule.

There is a sub-study to the main BICS study - that stratifies BICS participants into those with and without unrecognised heart disease based on blood biomarkers and echocardiogram to investigate whether any beneficial effect of bisoprolol is restricted to those with previously unrecognised heart disease.

\section{Methods}

\section{[9] Study setting}


Participants are being recruited from primary and secondary care sites across the UK. In primary care some General Practices are acting as recruitment sites, whereas others act as Participant Identification Centres (PICs) with identified participants being evaluated in other primary or secondary care recruitment sites. A list of the study sites can be found at https://w3.abdn.ac.uk/hsru/BICS/Public/Public/index.cshtml.

\section{[10] Eligibility criteria}

Patients will be enrolled if they meet all of the following criteria:

- Aged $\geq 40$ years.

- A smoking history of at least 10 pack years ([average number of cigarettes/day x years smoked]/20).

- An established predominant diagnosis of COPD (NICE Guideline definition: post bronchodilator $\mathrm{FEV}_{1}<80 \%$ predicted, $\left.\mathrm{FEV}_{1} / \mathrm{FVC}<0.7\right)^{1}$ receiving treatment as per local guidelines'. Patients with asthma-COPD overlap syndrome (ACOS) will also be eligible.

- A history of at least two exacerbations requiring treatment with antibiotics and/or oral corticosteroid use in the previous year, based on patient report OR a history of at least two exacerbations within 12 months of each other requiring treatment with antibiotics and/or oral corticosteroid since March 2019.

- Clinically stable with no COPD exacerbation for at least 4 weeks.

- Able to swallow study medication.

- Able and willing to give informed consent to participate.

- Able and willing to participate in the study procedures, complete study questionnaire.

- Able and willing to undergo spirometric assessment, able to perform an $\mathrm{FEV}_{1}$ manoeuvre as a minimum. During the COVID-19 pandemic, measurement of FEV ${ }_{1}$ is not required as part of the protocol, and therefore this inclusion criteria does not need to be met.

The main exclusion criteria are a diagnosis of asthma before the age of 40 years, a predominant respiratory disease other than COPD, use of beta-blockers, known intolerance to beta-blockers, use of drugs contraindicated with beta-blockers, ${ }^{31}$ resting heart rate $<60$ beats per min (bpm), systolic blood pressure $<100 \mathrm{mmHg}$, severe arterial occlusive disease, severe forms of Raynaud's syndrome, a history of psoriasis and conditions for which beta-blocker use is a guideline recommendation, e.g. heart failure. A detailed list all the exclusion criteria is included in the supplementary file. Concomitant use of drugs advised to be used with caution with beta-blockers is permitted. For women, current pregnancy or breastfeeding, or planned pregnancy during the study are exclusion criteria.

\section{[26a] Who will take informed consent?}

Consent is received by a suitably trained member of the research team at the recruitment site. 


\section{[26b] Additional consent provisions for collection and use of participant data and biological specimens}

Participants who wish to take part in the cardiac sub-study are asked to provide separate consent for this.

\section{[6b] Explanation for choice of comparator}

The use of placebo as comparator is acceptable because of clinical equipoise around the use of bisoprolol in people with COPD.

\section{[11a] Intervention description}

Participants will take either the cardio-selective beta-blocker bisoprolol (1.25mg tablets) or identical placebo for 52 weeks. Both are manufactured by Tiofarma B.V (Oud-Beijerland, Netherlands) and supplied by Mawdsley Brooks \& Co, (Doncaster, UK). Supplies of study drugs are couriered to the participants' homes. To ensure participant safety the starting dose for bisoprolol is one $1.25 \mathrm{mg}$ tablet taken orally daily and participants undergo a weekly dose titration regime (i.e. weekly increments of $1.25 \mathrm{mg} \rightarrow 2.5 \mathrm{mg} \rightarrow 3.75 \mathrm{mg} \rightarrow 5 \mathrm{mg}$ ) that results in final doses of $1.25 \mathrm{mg}$ once daily (od) ( $1 \mathrm{tab}), 2.50 \mathrm{mg}$ od (2 tabs), 3.75mg od (3 tabs), or $5 \mathrm{mg}$ od (4 tabs) depending on tolerance to bisoprolol up dosing. Participants allocated to placebo will undergo an identical dose-titration regime, with a final dose of 1,2 , 3 or 4 tablets a day. Participants complete the remainder of the 52-week treatment period on the final titrated dose. Figure 3 outlines the dose titration algorithm, decisions to increase, reduce or to fix on a dose during the titration period are determined by participant reports of intolerable side effects, heart rate, systolic blood pressure and self-reported changes to breathing. A computerised advisory titration algorithm is available and detailed in the supplementary file. Site staff can follow the titration advice or make an alternative decision about titration; this is documented as part of the case report form.

Following completion of the 52-week treatment period, participants will be weaned off study medication over the following 3 weeks (3-2-1 tablet od), cessation of study medication is confirmed by a telephone contact.

\section{[11b] Criteria for discontinuing or modifying allocated interventions}

During the 52-week treatment period the dose of study medication may be reduced at participant request or the development of possible adverse reactions. Participants may be withdrawn from treatment if consent for treatment is withdrawn, they develop unacceptable adverse reactions, they develop a condition for which beta-blockers are clinically indicated e.g. acute coronary syndrome, or a clinician wishes to prescribe a medication contraindicated with bisoprolol. Participants who withdraw consent are advised to wean down the study medication, participants withdrawn from treatment for other clinical reasons (i.e. adverse reactions) are advised to stop study treatment with immediate effect. Participants discontinuing study medication are invited to remain in the study and followed up in accordance with the trial protocol. 


\section{[11c] Strategies to improve adherence}

Adherence with study treatment is assessed at each titration assessment and the 26 and 52-week follow-up assessments by asking participants to estimate their adherence. ${ }^{32}$ Participant's preferences are incorporated into the titration process, so for example, if they do not wish to increase dose at any time, their preference is accommodated.

\section{[11d] Relevant concomitant care permitted or prohibited during the trial}

Participants remain on their usual COPD medications throughout the study period and clinicians are advised to manage participants in the usual manner subject to the caveats outlined above.

\section{[30] Post trial care}

At the end of the study, participants and their GPs will be informed of the study results and their allocation status. If at the end of their involvement a participant wishes to take bisoprolol, the participant's GP will be advised of this by letter. This letter will indicate that this would be off-label use of bisoprolol, that the patient may have been on placebo or bisoprolol and that dose-titration would be required.

\section{[12] Outcomes}

The primary outcome measure is the total number of exacerbations of COPD necessitating changes in management (minimum management change - use of oral corticosteroids or antibiotics) during the 52week treatment period, as reported by the participant. This clinically important outcome will be aggregated as mean events per year. The primary economic outcome measure is cost-per-Quality Adjusted Life Year (QALY) gained during the 52-week treatment period, this will be aggregated as a mean value.

The secondary outcomes will be quantified for the 52-week treatment period and are: number of participant reported COPD exacerbations requiring hospital admission; number of participant reported emergency hospital admissions (all causes); number of major adverse cardiovascular events (MACE); ${ }^{33}$ all-cause, respiratory and cardiac mortality; and utilisation of primary or secondary health care services for respiratory events. These outcomes will be expressed as a rate - number of events during the year of treatment. Additional secondary outcomes during the 52-week treatment period are: time to first exacerbation of COPD; post bronchodilator lung function ( $\left.F E V_{1}, F V C\right)$ (although because of COVID-19 this outcome will not be available for many participants); breathlessness using Baseline and Transition Dyspnoea Indices (BDI \& TDI); ${ }^{34}$ serious adverse events, adverse reactions; health related quality of life using EuroQoL 5D (EQ-5D-5L) Index, ${ }^{35}$ disease specific health status using the COPD Assessment Test (CAT); ${ }^{36}$ and modelled lifetime incremental cost per QALY.

\section{[13] Participant timeline}


Potential participants usually receive a postal invitation to participate that includes a participant information sheet and details on how to contact the local study team.

The participant timeline is outlined in figure 2. Participants are recruited and consented at a baseline face to face or telephone/video assessment. Participants are then reviewed at weekly telephone/video assessments for dose titration. The titration assessments are a minimum of one week apart, the usual time available (determined by first supply of study medication) for dose titration is seven weeks, this reflects 'real life' by accommodating participant's needs and wishes, however this period can be extended if required (for example if the participant has an exacerbation of their COPD during the titration period). Further follow-up telephone/video assessments take place at 26 and 52 weeks into the treatment period. After the 52-week treatment period participants are contacted by telephone after an appropriate number of weeks (one week per tablet) to confirm that they have weaned off the study medication.

Participants recruited in secondary care sites contributing to the cardiac sub-study are invited to participate in the sub-study during the titration phase, a separate consent is obtained covering echocardiography and collection and analysis of blood samples.

\section{[14] Sample size}

The multicentre Evaluation of COPD Longitudinally to Identify Predictive Surrogate Endpoints (ECLIPSE) study reported the frequency of COPD exacerbation in 2138 patients. ${ }^{37}$ For patients identical to our target population ( $\geq 2$ self-reported COPD exacerbations in a year requiring antibiotics and/or OCSs), the mean (SD) number of COPD exacerbations within the subsequent one year was 2.22 (1.86). The Cardiac Insufficiency Bisoprolol Study II (CIBIS -II) reported that about $15 \%$ of participants stopped taking study medication in a trial of bisoprolol vs placebo. ${ }^{26}$

Assuming a similar rate in the placebo arm 669 participants are needed in each arm of the trial to detect a clinically important reduction in COPD exacerbations of $15 \%$ (i.e. from an average of 2.22 to 1.89) with $90 \%$ power at the two-sided $5 \%$ significance level. Allowing for an estimated $15 \%$ withdrawal from study treatment 787 participants are required in each study group (i.e. 1,574 in total).

\section{[15] Recruitment}

Potential participants will be recruited from both primary and secondary care sites across the UK, the aim being to recruit the majority of participants $(>50 \%)$ in primary care sites. Recruitment strategies will differ between centres depending on local geographic and NHS organisational factors.

\section{Primary care}

Recruitment in General Practices will be conducted in conjunction with the NIHR Clinical Research Network (CRN) in England and the NHS Research Scotland Primary Care Network in Scotland. In primary care, potential participants are identified from General Practice databases that are searched for the major inclusion and exclusion criteria. Preliminary lists of potential participants are reviewed by a medical 
practitioner. In some centres community based respiratory services e.g. pulmonary rehabilitation, respiratory nurses are available and are used to identify potential participants. Recruitment in primary care is supplemented by posters located in General Practice waiting areas and Community Pharmacies.

\section{Secondary care}

In secondary care, potential participants are identified from patients attending hospital Respiratory OutPatient clinics, spirometry services, smoking cessation services and early supported discharge services. Some trial centres also have access to Volunteer Databases/Registries. Potential participants in the cardiac sub-study are participants who have been recruited in secondary care sites contributing to the sub-study.

\section{[16a] Sequence generation}

Participants will be enrolled and consented by a member of the site research team who then use a computerised web based randomisation service to allocate the participant to a treatment group. The randomisation service is administered by the Centre for Healthcare Randomised Trials (CHaRT), University of Aberdeen. The randomisation is stratified by trial centre (or area for primary care sites), and recruitment setting (primary or secondary care) and participants are randomised with equal probability $(1: 1)$ to the intervention and control groups. The random allocation sequence has been generated using permuted blocks to provide randomly generated blocks of entries of varying sizes permuted for each combination of region and recruitment setting.

\section{[16b] Concealment mechanism}

The web-based randomisation system ensures allocation concealment.

\section{[16c] Implementation}

The randomisation system is embedded in the trial website. A member of the research team at the recruitment site randomises a participant after consent.

\section{[17a] Who will be blinded}

To ensure double blinding, bisoprolol and placebo tablets are identical in appearance, taste, touch and smell and dispensed in identically labelled containers. All trial participants, care providers, outcome assessors, trials managers, data analysts remain blinded to allocation status until database lockdown. Unblinding of allocation status is permitted to enable treatment of severe adverse event/s, overdose, or to enable SUSAR reporting. The trial database includes an unblinding function with access limited to those permitted to unblind in and out of office hours. At the end of the 12 month follow-up period, if a participant requests information as to their allocation within the trial, for example to plan future treatment, the Sponsor can agree to unblinding. In such cases, the $\mathrm{Cl}$ and trial office team remain blind to the treatment allocation. Where possible the site team also remain blind to the treatment allocation.

\section{[17b] Procedure for unblinding}


All sites have access to an automated telephone unblinding system that can be used to unblind a participant if necessary. In addition, participants are given an emergency card that includes an emergency contact number. The $\mathrm{Cl}$ and their team have access to a web-based automated unblinding system in addition to the automated telephone unblinding system).

\section{Data collection and management \\ [18a] Plans for assessment and collection of outcomes}

The schedule for data collection is outlined in figure 2. The following data are collected:

\section{Drug history}

Regular use of prescription drugs is recorded at recruitment and the 26 and 52 week assessments.

\section{Smoking history}

Smoking history (age commenced, age ceased, average cigarettes smoked per day) is recorded at recruitment, and at the 26 and 52 week assessments. Pack year consumption is computed at recruitment.

\section{Height and weight}

Height and weight are measured at recruitment.

\section{Heart rate and blood pressure}

Resting heart rate and blood pressure are measured at recruitment and the dose titration assessments at week one, two, three, four and the 26 and 52 week assessments.

\section{Number of COPD exacerbations}

The primary outcome measure of the total number COPD exacerbations requiring antibiotics/oral corticosteroids whilst on study medication will be ascertained by asking the participants at the 26 and 52 week assessments. Participants are encouraged to record any exacerbations on a provided 'reminder card' and to have this available during their follow-up assessments. The American Thoracic Society/European Respiratory Society guideline definition of COPD exacerbation will be used: a worsening of patient's dyspnoea, cough and/or sputum beyond day-to-day variability sufficient to warrant a change in management. ${ }^{38}$ The minimum management change will be treatment with antibiotics and/or oral corticosteroids. A minimum of two weeks between consecutive hospitalisations/start of new therapy is necessary to consider events as separate. A modified ATS/ERS operational classification of exacerbation severity will be used for each exacerbation: Level I Increased use of short acting $\beta 2$ agonist; Level II, use of oral corticosteroids or antibiotics; Level III, care by services to prevent hospitalisation; Level IV, admitted to hospital. ${ }^{38}$

\section{Hospital admissions}


The number of unscheduled hospital admissions whilst on study medication is ascertained at the 26-and 52-week assessments. Emergency COPD admissions will also be identified.

\section{Major adverse cardiovascular events (MACE)}

MACE as defined by cardiovascular death, hospitalisation for myocardial infarction, heart failure, or stroke, percutaneous coronary intervention or coronary artery bypass grafting, will be ascertained at the 26- and 52-week assessments. ${ }^{33}$

\section{Health related quality of life}

Health related quality of life data will be captured at recruitment, 26 and 52 weeks using EuroQoL 5D-5L (EQ-5D-5L) Index that has been used widely in studies of COPD. ${ }^{35}$ EQ-5D-5L was developed as a utility questionnaire and addresses mobility, self-care, usual activities, pain/discomfort and anxiety/depression. The completed instrument can be translated into quality of life utilities suitable for calculation of QALYs through the published United Kingdom tariffs. ${ }^{39}$

\section{Disease related health status}

Disease related health status will be ascertained at recruitment and at the 26- and 52-week assessments by questionnaire using the COPD Assessment Test (CAT). ${ }^{36}$ The CAT is an 8-item unidimensional measure of health status impairment in COPD. The score ranges from 0-40; it correlates very closely with health status measured using the St George Respiratory Questionnaire and is reliable and responsive. The CAT score is preferred since it provides a more comprehensive assessment of the symptomatic impact of COPD. ${ }^{40,41}$

The Baseline Dyspnoea Index (BDI) questionnaire will be administered at the recruitment, assessment and the Transitional Dyspnoea Index (TDI) will be administered at the 26- and 52-week assessments. ${ }^{42}$ $\mathrm{BDI}$ and TDI were developed in order to obtain a comprehensive understanding of patients' severity of breathlessness and are based on three components: functional impairment; magnitude of task; and magnitude of effort. BDI is a discriminative instrument used to quantify the severity of dyspnoea at an initial or baseline state, whereas TDI is an evaluative instrument used to quantify the changes in dyspnoea from the initial or baseline state. ${ }^{34}$

\section{Post bronchodilator lung function}

Post bronchodilator lung function will be measured by spirometry performed to ATS/ERS standards, at recruitment, titration visits (weeks one, two, three and four), and 26 and 52 weeks. ${ }^{43}$ However, because of COVID-19 it will not be possible for spirometry to be performed and this outcome will not be available for many participants.

\section{Health care utilisation}

Health care utilisation during the previous 6 months is recorded at recruitment and at the 26- and 52-week assessments using a modified version of the Client Service Receipt Inventory (CSRI). ${ }^{44}$ The CSRI is a 
research questionnaire for retrospectively collecting cost related information about participant's use of health and social care services.

\section{Adverse reactions and serious adverse events}

Adverse reactions and serious adverse events are recorded at each titration assessment, the 26- and 52week assessments and the end of weaning 'phone call. Participants are notified of recognised adverse reactions and encouraged to contact the local study centre if they experience these.

\section{Mortality}

Deaths during the follow-up period are recorded and reported as Serious Adverse Events.

\section{Adherence}

Adherence with study treatment is assessed at each assessment by asking participants to estimate their adherence.

\section{Echocardiography}

Participants in the cardiac sub-study undergo echocardiography as early as possible during the titration period subject to logistics and the processes of informed consent. Echocardiography is performed according to standard protocols on GE or Philips systems, inclusive of diastolic function, speckle and tissue Doppler imaging for offline analysis. ${ }^{45}$ Apical 2,3 and 4 chamber views are acquired for accurate computation of ejection fraction, atrial volumes, left ventricular wall thickness and estimated pulmonary artery pressure. The images are promptly sent to the University of Aberdeen, Department of Cardiology for analysis. Any significant or clinically relevant findings that are likely to significantly impact on the patient's health or future prognosis will be provided to the participant's GP (with the participant's agreement).

\section{Blood assays}

Participants in the cardiac sub-study have a venous blood sample taken as early as possible during the titration period and at the final 52-week assessment. These samples will be assayed for high-sensitivity cardiac troponin I (hs-cTnl), N-terminal pro-Brain Natriuretic Peptide (NT-proBNP), Galectin and highsensitivity C-reactive protein (hs-CRP) by laboratories at the Universities of Dundee and Edinburgh. HscTnl is a biomarker of myocardial damage and NT-proBNP is released in response to changes in intra cardiac pressure. ${ }^{44}$

\section{[18b] Plans to promote retention and complete follow-up}

To promote retention, participants unable to take part in the 26- and 52-week assessments will be sent the questionnaire to complete at home. Participants who cease taking the study medication will be invited to participate in the 26- and 52-week assessments. For participants who do not participate in the 52-week assessment and who do not complete the questionnaire, attempts will be made to identify the number of 
exacerbations in the appropriate time period by examining GP records, if possible MACE outcomes will be determined at the same time.

\section{[19] Data management}

At baseline and at follow-up assessments, study data can be entered directly into the study website maintained by CHaRT and held on a secure server, the study website has a full audit trail. Sites that directly enter data into the website are encouraged to print or save a copy of the electronic data in order to maintain a copy of the data independent of that held by the Sponsor. Study data collected on hard copy case report forms are subsequently entered into the study website. The central trials team monitor data entry and ensure that missing or implausible data are addressed as soon as possible after detection. All study documentation will be archived for at least 25 years after publication of the study data.

\section{[27] Confidentiality}

All Investigators and study site staff involved comply with the requirements of GCP and the UK Data Protection Acts 2018. Data will be stored for at least 25 years after publication. Publications will not contain any personal data that could allow identification of individual participants.

\section{[33] Plans for collection, laboratory evaluation and storage of biological specimens for genetic or molecular analysis in this trial/future use}

Details about the collection of the blood samples and echocardiography are described in section 18a above. Any residual material will be retained in an approved tissue bank.

\section{[20a] Statistical Methods for primary and secondary outcomes}

Statistical analyses will be conducted in accordance with the intention to treat principle with a per protocol analysis performed as a sensitivity. The per protocol analysis will exclude participants who were not compliant (at less than 70\%) with their study medication. All analyses will be governed by a comprehensive statistical analysis plan that will be finalised before the data lock.

The primary clinical outcome of number of COPD exacerbations will be compared between randomised groups using negative binomial regression with length of time in the study as an offset. Estimates will be adjusted for centre and other baseline covariates known to be related to outcome (e.g. age, smoking, COPD hospitalisations in year prior to study, medications). An over dispersion parameter will be used to adjust for between patient variability. To assess the impact of death (estimated at around $6 \%$ ) ${ }^{46}$ a sensitivity analysis will be undertaken by excluding those subjects who have died. For participants that are lost to follow up (estimated to be around 15\%), ${ }^{46}$ their information will be included in the statistical models up to the point that they are lost to follow up. Sensitivity analyses will be undertaken using 
multiple imputation (assuming data are missing at random), and, if necessary, and the data permit, specify the mechanism of missing data via a pattern mixture model assuming informative missingness.

The secondary outcomes - total number of COPD exacerbations requiring hospital admission and total number of emergency hospital admissions (all causes) - will be analysed in the same way as for the primary outcome. Mixed effects models will be used to compare the secondary outcomes, CAT, EQ5D-5L, $\mathrm{FEV}_{1}$ and $\mathrm{FVC}$, by randomisation group adjusted for centre, patient characteristics and/or baseline clinical variables. All-cause mortality rate and time to first COPD exacerbation, will be compared between randomised group using a log-rank test and Kaplan-Meier survival curves. Adjustment for potential covariates will be undertaken using Cox proportional hazards regression. Major adverse cardiovascular events (MACE) in randomisation groups will be compared using the chi-square test.

A National Health Service perspective will be adopted in keeping with the NICE reference case for health technology assessments. ${ }^{47}$ The two-stage economic evaluation will comprise an analysis of treatment cost-effectiveness within trial period and then extrapolated to lifetime using cost-effectiveness modelling The within trial analysis will use health care resource use data (translated to a cost-per-patient using unit costs standard reference sources), the exacerbation rate associated with the treatment groups, and the quality of life effects estimated from the EQ-5D-5L combined with length of life to calculate QALYs. Nonparametric bootstrapping will be used to capture sampling uncertainty in the observed data and results will be presented as cost-per-exacerbation avoided and cost-per-QALY gained within the trial period. The extrapolation analysis will make use of regression estimates of exacerbation on cost and quality of life from the trial, as well as previously published models of COPD, to guide the extrapolation to patient lifetimes. In addition to sampling uncertainty, extensive sensitivity, analysis will be performed to understand the importance of alternative modelling assumptions for the extrapolated results.

\section{[21b] interim analyses}

There will be no interim analyses.

\section{[20b] Methods for additional analysis}

Sub-group analysis of participants with clinically diagnosed heart disease to determine whether any beneficial effects of bisoprolol are limited to those with clinically diagnosed heart disease. Additional sub-group analysis of the primary outcome are planned (including by sex and smoking status).

\section{[20c] Methods in analysis to handle protocol non-adherence and any statistical methods to handle missing data}

The primary analysis will use the intention to treat principle. A per protocol analysis will include those patients who took $70 \%$ or more of their study medication. Sensitivity analysis to handle missing data are described above [20a]. 


\section{[31c] Plans to give access to the full protocol, participant level-data and statistical code}

The full protocol is included as supplementary material. Requests for access to participant level data and/or the statistical code should be made in writing to the Chief Investigator Graham Devereux (graham.devereux@lstmed.ac.uk). Data will be provided after review, approval by investigators and a data transfer agreement has been signed.

\section{[5d] Composition of the coordinating centre and trial steering committee}

The immediate trial team based in the coordinating centre $(\mathrm{Cl}$, trial manager, data coordinator) meet weekly initially. The frequency of these meetings is likely to reduce as the trial progresses, but can revert to weekly meetings as and when required. On a monthly basis, the immediate team is joined by a wider team (statistician, health economist). A Project Management Group (PMG) and Trial Steering Group (TSC) oversee the project. The PMG meets approximately every three months and comprises the Chief Investigators, grant holders (including clinical, methodological, statistical, health economic and qualitative expertise) and the trial office staff. The TSC meet approximately every six months and includes an independent chair, clinical and methodological expertise, and lay representatives.

\section{[21a] Composition of the data monitoring committee, its role and reporting structure}

The independent Data Monitoring Committee (DMC) comprises an independent chair and independent members with clinical, statistical and methodological expertise. The DMC meets every 6 months or more frequently if required. The DMC is independent of the sponsor and competing interests and reports to the chair of the Trial Steering Committee.

\section{[22] Adverse event reporting and harms.}

The trial complies with the United Kingdom National Health Service National Research Ethics Service guidelines for reporting adverse events. ${ }^{48}$ Adverse reactions (ARs) and serious adverse events (SAEs) are recorded from the time a participant consents to join the study until the end of the weaning period after the 52-week follow-up. Participants who withdraw from taking the study drug during the 52-week treatment period have ARs and SAEs recorded from consent until 28 days after ceasing study medication. Exacerbations of COPD or hospital admissions as a consequence of exacerbations of COPD are not considered as ARs or SAEs because they are primary and secondary outcomes for the trial. The Reference Safety Information used in the assessment of SAEs/ARs is based on the SmPC for bisoprolol and is located on the study website. ${ }^{31}$

\section{[23] Auditing}


The trial office monitor aspects of the study on an ongoing basis as described in the study monitoring plan prepared at the outset of the study. The trial is also monitored and audited by the Sponsor. Individual sites may be monitored by their local Research and Development department.

\section{[25] Plans for communicating important protocol amendments to relevant parties}

Changes to the protocol require the trial office to seek permission from the funder, sponsor, REC and NHS R\&D offices.

\section{[31a] Dissemination policy}

A summary of the study findings will be sent to surviving trial participants, their GPs and investigators. The clinical study report will be used for publication and presentation at scientific meetings and at patient and clinical interest group events. The publication policy is included in the protocol supplementary file.

\section{Discussion}

There is a pressing need to identify interventions that reduce the impact of COPD, a common disease that continues to be associated with high morbidity, mortality and healthcare costs. BICS is a pragmatic randomised double-blind placebo-controlled trial that tests the hypothesis that adding the beta-blocker bisoprolol to routine COPD treatment reduces the rate of exacerbation. The cardiac sub-study tests the hypothesis that any beneficial effect of bisoprolol is restricted to those with unrecognised heart disease.

Recruitment to the BICS trial was paused in March 2020 due to the COVID-19 pandemic and remained paused until July 2021 when the Sponsor and Funder agreed that the study could re-open to recruitment. As part of the plans for re-opening, a number of revisions were made to the protocol.

Firstly, we reduced the number of face to face assessments. When the study first opened to recruitment in 2018, there were up to 7 face-to-face assessments (baseline, four titration visits, follow-up at 26 and 52 weeks). In response to COVID-19 the number of face-to-face assessments for the main BICS study was reduced to one baseline assessment; although if the site or participant prefer to carry out the baseline assessments remotely (by telephone or videocall), this is permitted. Those participants who wish to take part in the sub-study will have two additional face-to-face assessments, one soon after recruitment for the blood samples and echocardiogram, and one around the 52-week follow-up for blood samples.

Secondly, we amended the inclusion criteria. When the study first opened to recruitment, one of the inclusion criteria was "at least two exacerbations requiring treatment with antibiotics and/or oral corticosteroid use in the previous year, based on patient report" and this was revised so that the two exacerbations could have occurred since March 2019, providing they were within 12 months of each other. The number of exacerbations experienced by people with COPD declined during the COVID-19 pandemic probably because of COVID-19 mitigation measures e.g. shielding, social distancing measures, and face coverings ${ }^{49}$. The ECLIPSE study has demonstrated that the frequent-exacerbation phenotype is 
relatively stable over a three year period and the strongest predictor for further exacerbations is a history of exacerbations ${ }^{37}$. The change in inclusion criteria regarding the timing of previous exacerbations enables the identification of the people who remained at high risk of exacerbation even during the time of reduced exacerbation risk. We also amended the inclusion criteria to remove the requirement for spirometry (which was classed as an aerosol generating procedure and thus restricted within the NHS). When the study first opened to recruitment, the use of current or historical evidence of $F E V_{1} / F V C<0.7$ was permitted but $\mathrm{FEV}_{1}<80 \%$ predicted needed to be demonstrated at baseline. The inclusion criteria were amended so that both $\mathrm{FEV}_{1} / \mathrm{FVC}<0.7$ and $\mathrm{FEV}_{1}<80 \%$ predicted are based on historical readings recorded in the medical records.

Thirdly, we amended the titration schedule within the study. When the study first opened to recruitment, the titration schedule included $\mathrm{FEV}_{1}$. Again, because of the limitations on spirometry in the NHS, the titration schedule was revised so that $\mathrm{FEV}_{1}$ was replaced with participant self-report of their breathing with a particular focus on deterioration since the study medication was started or the dose of study medication was increased.

Beta-blockers are predominantly used in the management of cardiovascular conditions e.g hypertension, heart failure, however it has been observed that beta-blocker use in people with COPD is associated with a reduced risk of exacerbation. ${ }^{18-24}$ A systematic review of observational cohort studies of beta-blocker use for cardiovascular conditions demonstrated that beta-blocker use in people with COPD was associated with reduced mortality and exacerbation rate. ${ }^{18}$ The BICS trial is one of the first to investigate whether commencing people on a beta-blocker for their COPD is beneficial. The cardiac sub-study will investigate whether any beneficial effect of bisoprolol is limited to those people with COPD with unrecognised heart disease as determined by risk stratification based on echocardiography and blood concentrations of Galectin, hs-cTnl, hs-CRP and NTproBNP. 45

The sample size for current study was based on the ECLIPSE study reporting that for people with COPD with two or more exacerbations in a year the mean (SD) number of COPD exacerbations within the subsequent one year was 2.22 (1.86). In our trial of low dose theophylline in COPD (TWICS), for people with COPD with two or more exacerbations in the previous year the mean $(95 \% \mathrm{Cl})$ number of exacerbations in the subsequent year was $2.23(2.09,2.37)$. The TWICS study also confirmed reports that patient recall of COPD exacerbations is reliable over a year with $79 \%$ concordance between participant and GP clinical records of exacerbation in a validation exercise. ${ }^{46,50}$

BICS is one of several trials investigating the role of beta-blockers in people with COPD. BLOCK COPD was a multicentre randomised double blind placebo controlled trial of metoprolol in people with moderate/severe COPD ( $\mathrm{FEV}_{1}<80 \%$ predicted) conducted in the United States (protocol NCT02587351). ${ }^{51}$ Participants were randomised 1:1 to extended release metoprolol succinate or placebo with a 6 week dose titration phase resulting in final doses of $25 \mathrm{mg}, 50 \mathrm{mg}$ or $100 \mathrm{mg}$ od, the total treatment period was 52 weeks. The inclusion criteria included $\geq 1$ exacerbation in the previous year treated with antibiotics 
and/or systemic corticosteroids or, to be using, or have been prescribed supplemental home oxygen for at least 12 hours a day. The primary outcome was the time to the first moderate/severe COPD exacerbation, the study sample size of 1028 was powered to detect a $15 \%$ reduction in the probability of an exacerbation in the year treatment period. This trial was terminated prematurely after recruitment of 532 participants because of futility with respect to the primary end point and safety concerns. There was no significant between group difference in the median time to first exacerbation, (202 days metoprolol, 222 days placebo group). Although metoprolol was associated with a higher risk of exacerbation leading to hospitalization (hazard ratio, $1.91 ; 95 \% \mathrm{Cl}, 1.29$ to 2.83), in the year prior to commencing study drug, the metoprolol group were more likely to have a COPD exacerbation leading to emergency department visit or hospitalization (62.7\% vs 50.4\%, $\mathrm{p}=0.004$ ). The BICS trial differs from BLOCK COPD in several important respects. In BICS the beta-blocker bisoprolol is being tested because, unlike metoprolol, it is licensed for use in heart failure in the UK and has a higher beta1:beta2 receptor selectivity ratio (14:1) than metoprolol (2:1)..$^{52}$ In BLOCK COPD potential participants had an electrocardiograph (ECG) recording and were rendered ineligible by prespecified ECG abnormalities. The pragmatic design of BICS replicates the routine practice of commencing beta-blockers in UK primary care settings without an ECG, thus when compared with BLOCK COPD the participants in BICS are likely to represent COPD patients in the UK NHS, to have more unrecognised heart disease and benefit from beta-blocker therapy. A study of bisoprolol in preventing adverse cardiac events in COPD in Australia and New Zealand (protocol NCT03917914) commenced recruitment in June 2020. In this multicentre randomised double-blind placebo controlled trial 1164 people with $\mathrm{COPD}$ ( $\mathrm{FEV}_{1}<70 \%$ predicted) with $\geq 1$ exacerbation in the previous year will be randomised 1:1 to bisoprolol or placebo, the treatment period is two years and the doses of bisoprolol will be $1.25 \mathrm{mg}, 2.5 \mathrm{mg}$ or $5 \mathrm{mg}$ od. The primary outcomes include all cause mortality, hospitalisation for COPD exacerbation, hospitalisation for primary cardiac cause, and major adverse cardiovascular events. A single arm open label trial of bisoprolol in people with moderate/severe COPD ( $\mathrm{FEV}_{1}<80 \%$ predicted) and hypertension conducted in Japan (protocol UMIN000024712) appears to have ceased recruitment. ${ }^{53} \mathrm{~A}$ total of 35 participants were to be recruited and commenced on bisoprolol $1.25 \mathrm{mg}$ od, increasing monthly to $2.5 \mathrm{mg}$ od and $5 \mathrm{mg}$ od if tolerated, the primary outcome was the rate of moderate-to-severe COPD exacerbation during the two year treatment period. The study sample size was powered to detect a $25 \%$ reduction in rate of exacerbation. BRONCHOLE is a multicentre randomised open label controlled trial of metoprolol in people with COPD being conducted in the Sweden (protocol NCT03566667) ${ }^{54}$. All participants will be randomised 1:1 to metoprolol or standard care, the target metoprolol dose is $100 \mathrm{mg}$ od and the treatment period is 52 weeks. There are no exacerbation inclusion criteria and potential participants are excluded if their records contain any International Classification of Diseases (ICD) codes for cardiovascular diseases. The primary outcome is a composite of all cause mortality, COPD exacerbations and cardiovascular events, the study sample size of 1700 is powered to detect a $25 \%$ reduction in the primary outcome, however this outcome is dominated by exacerbations. This trial is due to finish recruitment in December 2021.

It is almost certain that a substantial proportion of the participants in BICS will have severe lung disease and will have limited exercise tolerance. When the study first opened to recruitment, allowances were 
made in the trial design to facilitate participation by this group of patients: at site discretion, participants could be recruited and dose titrated home using portable spirometers and sphygmomanometers and those unable to attend for follow up assessment visits would be assessed by telephone review and postal collection of quality of life questionnaires. We hope that the revisions made to the protocol to allow restart have made the study even more accessible to this group of patients. Study medication is be couriered directly to the homes of participants, thus avoiding travel to study centres to collect supplies.

The dose each participant takes for the duration of the study is determined by titrating the dose of study drug during the first 4 weeks of the treatment period, however to increase flexibility and to replicate 'real life' there is sufficient medication in the first batch of medication for the dose titration to be conducted over 7 weeks. The study drug is provided as $1.25 \mathrm{mg}$ bisoprolol tablets, the dose titration results in final doses of $1,2,3$ or 4 tablets a day. A single tablet was chosen to avoid the complications of different tablet strengths and doses. The dose titration schedule is a conservative interpretation of the 'start low, go slow' advice provided in heart failure guidelines designed for use by appropriately trained nurses in primary care settings. ${ }^{29-30}$ Decisions to increase, reduce or to fix on a dose during the titration period are determined by participant reports of intolerable side effects, heart rate, systolic blood pressure and changes in breathing (replaced the pre COVID-19 lung function criterion). The algorithm is included in the study website and requires research staff to enter these parameters and the programme provides an advisory recommendation, at all times clinicians are free to ignore the advice and to make their own clinical decision.

Bisoprolol is a relatively cheap drug familiar to many clinicians whose use is established in people with heart failure and COPD. The demonstration that the addition of bisoprolol to routine COPD treatment reduces the likelihood of exacerbation will be relevant not only to patients and clinicians but also to healthcare providers, both in the UK, and globally.

\section{Trial Status}

The first subject was recruited on 16/10/2018. The trial was paused to recruitment in March 2020 due to the COVID pandemic. It remained paused to recruitment until July 2021 when the Sponsor and Funder agreed that the study could re-open to recruitment. BICS is currently recruiting patients.

\section{Abbreviations}

BDI: Baseline dyspnoea index

BICS: Bisoprolol in COPD study

CAT: COPD Assessment Test.

CHaRT: Centre for Healthcare Randomised Trials. 
COPD: Chronic Obstructive Pulmonary Disease.

$\mathrm{FEV}_{1}$ forced expiratory volume in first second.

FVC forced vital capacity.

hs-cTnl: high sensitivity cardiac troponin I.

NICE: National Institute for Health and Care excellence.

NTproBNP: N-terminal pro-Brain Natriuretic Peptide

QALY: Quality Adjusted Life Year.

TDI: Transition dyspnoea index

£: United Kingdom pound sterling

\section{Declarations}

\section{Acknowledgments}

We are grateful for the secretarial and data co-ordination support from Janice Cruden. We would like to acknowledge the principal investigators and staff based in trial recruitment sites across the country, the Clinical Research Networks and staff in the Clinical Trials Pharmacy, and the support of the Trial Steering and Data Monitoring Committees. In particular, we have been grateful for the input to the Trial Steering Committee from two lay representatives, Mr Alister Laird and Mr Dave Bertin.

\section{[31b] Authors contributions}

$B L$ and GD conceived the study, led the proposal and protocol development. SC, AB, KC, RC, GC, ADS, SF, SG, JH, AL, WM, KM, NMcM, AM, JN, DP, PS, JV, PW, JW and AW contributed to the study design and to the development and implementation of the protocol. BL, GD and DD conceived the cardiac sub-study. HA, CCL, NLM, and MP contributed to the sub-study design and development of the protocol. All authors read and approved the final manuscript.

\section{[4] Funding}

This project was funded by the National Institute for Health Research, Health Technology Assessment Programme (project number 15/130/20). The cardiac sub-study is funded by British Heart Foundation (BHF) Project Grant no. PG/17/64/33205. This report presents independent research commissioned by the National Institute for Health Research (NIHR). The views and opinions expressed by authors in this publication are those of the authors and do not necessarily reflect those of the NHS, the NIHR, MRC, CCF, NETSCC, the Health Technology Assessment programme or the Department of Health. 
[29] Availability of data and materials

At the end of the trial, the statistician and the health economist will have access to the full dataset to permit analysis.

\section{[24] Ethics approval and consent to participate}

The trial has received a favourable opinion from Scotland A Research Ethics Committee (ref 18/SS/0033, 22/05/2018) and the Medicines and Healthcare products Regulatory Agency (MHRA) (EudraCT 2017002779-24, CTA 21583/0222/001-0001, 12/06/2018). All participants provide written informed consent.

\section{[32] Consent for publication}

A copy of the participant information leaflet and consent form are available as supplementary material.

\section{[28] Competing interests}

The study is funded by the NIHR Health Technology Assessment. The Chief Investigator, study grant holders and protocol authors have no financial or competing interests.

\section{References}

1 Chronic obstructive pulmonary disease in over 16s: diagnosis and management. 2018 https://www.nice.org.uk/guidance/ng115

2. WHO. Global surveillance, prevention and control of chronic respiratory diseases: a comprehensive approach. 2007. https://www.who.int/gard/publications/GARD_Manual/en/

3. Adeloye D, Chua S, Lee C, Basquill C, Papana A, Theodoratou E, Nair H, Gasevic D, Sridhar D, Campbell H, Chan KY, Sheikh A, Rudan I. Global and regional estimates of COPD prevalence: Systematic review and meta-analysis. J Glob Health. 2015;5:020415. doi: 10.7189/jogh.05-020415.

4. Mathers C, Loncar D. Projections of global mortality and burden of disease from 2002 to 2030 . PLoS Medicine. 2006;3: e442.

5. British Lung Foundation (2015). Health statistics. http://statistics.blf.org.uk/copd

6. Mannino DM, Thorn D, Swensen A, Holguin F. Prevalence and outcomes of diabetes, hypertension and cardiovascular disease in COPD. Eur Respir J 2008; 32: 962-969.

7. Holguin F, Folch E, Redd SC, Mannino DM. Comorbidity and mortality in COPD-related hospitalizations in the United States, 1979 to 2001. Chest 2005; 128: 2005-2011.

8. Antonelli-Incalzi R, Fuso L, DeRosa M, Forastiere F, Rapiti E, Nardecchia B, Pistelli R. Co-morbidity contributes to predict mortality of patients with chronic obstructive pulmonary disease. Eur Respir J 1997; 
10: $2794-2800$.

9. Sin DD, Man JP, Man SF. The risk of osteoporosis in Caucasian men and women with obstructive airways disease. Am J Med 2003; 114: 10-14.

10. Di Marco F, Verga M, Reggente M, Maria Casanova F, Santus P, Blasi F, Allegra L, Centanni S. Anxiety and depression in COPD patients: the roles of gender and disease severity. Respir Med 2006; 100: 17671774.

11. Rutten FH, Cramer MJ, Grobbee DE, Sachs AP, Kirkels JH, Lammers JW, Hoes AW. Unrecognized heart failure in elderly patients with stable chronic obstructive pulmonary disease. Eur Heart J 2005;26:18871894.

12. Macchia A, Rodriguez Moncalvo JJ, Kleinert M, Comignani PD, Gimeno G, Arakaki D, Laffaye N, Fuselli JJ, Massolin HP, Gambarte J, Romero M, Tognoni G. Unrecognised ventricular dysfunction in COPD. Eur Respir J 2012;39:51-58.

13. Donaldson GC, Seemungal TA, Bhowmik A, Wedzicha JA. Relationship between exacerbation frequency and lung function decline in chronic obstructive pulmonary disease. Thorax 2002;57:847-852.

14. Donaldson GC, Wilkinson TM, Hurst JR Perera WR, Wedzicha JA. Exacerbations and time spent outdoors in chronic obstructive pulmonary disease. Am J Resp Crit Care Med 2005;171:446-452.

15. Seemungal TA, Donaldson GC, Paul EA Bestall JC, Jeffries DJ, Wedzicha JA. Effect of exacerbation on quality of life in patients with chronic obstructive pulmonary disease. Am J Resp Crit Care Med 1998;157:1418-1422.

16. Soler-Cataluna JJ, Martinez-Garcia MA, Roman Sanchez P et al. Severe acute exacerbations and mortality in patients with chronic obstructive pulmonary disease. Thorax 2005;60:925-931.

17. Britton M. The burden of COPD in the U.K.: results from the Confronting COPD survey. Respir Med 2003;97:S71-79.

18. Du Q, Sun Y, Ding N, Chen Y. Beta-Blockers Reduced the Risk of Mortality and Exacerbation in patients with COPD: A Meta-Analysis of Observational Studies. PLoS ONE 2014; 9(11): e113048.

doi:10.1371/journal.pone.0113048

19. Rutten FH, Zuithoff NP, Hak E, Grobbee DE, Hoes AW. Beta-blockers may reduce mortality and risk of exacerbations in patients with chronic obstructive pulmonary disease. Arch Intern Med. 2010;170:880887.

20. Bhatt SP, Wells JM, Kinney GL, Washko GR Jr, Budoff M, Kim YI, Bailey WC, Nath H, Hokanson JE, Silverman EK, Crapo J, Dransfield MT. $\beta$-Blockers are associated with a reduction in COPD exacerbations. Thorax 2016;71:8-14. 
21. Gottlieb SS, McCarter RJ, Vogel RA. Effect of beta-blockade on mortality among high-risk and low-risk patients after myocardial infarction. New Eng J Med. 1998;339(8):489-497.

22. Au DH, Bryson CL, Fan VS, Udris EM, Curtis JR, McDonell MB, Fihn SD.. Beta-blockers as single-agent therapy for hypertension and the risk of mortality among patients with chronic obstructive pulmonary disease. Am J Med. 2004;117(12):925-931.

23. Short PM, Lipworth SI, Elder DH, Schembri S, Lipworth BJ. Effect of beta blockers in treatment of chronic obstructive pulmonary disease: a retrospective cohort study. Br Med J. 2011;342:d2549.

24. Quint JK, Herrett E, Bhaskaran K, Timmis A, Hemingway H, Wedzicha JA, Smeeth L. Effect of beta blockers on mortality after myocardial infarction in adults with COPD: population based cohort study of UK electronic healthcare records. Br Med J. 2013;347:f6650.

25. Hawkins NM, Petrie MC, Macdonald MR, Jhund PS, Fabbri LM, Wikstrand J, McMurray JJ. Heart failure and chronic obstructive pulmonary disease the quandary of Beta-blockers and Beta-agonists. .J Am Coll Cardiol 2011; 57:2127-2138.

26. CIBIS-II Investigators and Committees. The Cardiac Insufficiency Bisoprolol Study II (CIBIS-II): a randomised trial. Lancet 1999; 353: 9-13

27. British Thoracic Society/Scottish Intercollegiate Guidelines Network. British guideline on the management of asthma (2019). https://www.brit-thoracic.org.uk/qualityimprovement/guidelines/asthma/

28. Salpeter SR, Ormiston TM, Salpeter EE. Cardioselective beta-blockers for chronic obstructive pulmonary disease. Cochrane Database of Systematic Reviews 2005, Issue 4. Art. No.: CD003566. DOI: 10.1002/14651858.CD003566.pub2

29. Chronic heart failure in adults: management. Clinical guideline 2010. https://www.nice.org.uk/guidance/cg108

30. Scottish Intercollegiate Guidelines Network (SIGN). Management of chronic heart failure. Edinburgh: SIGN; 2016. https://www.sign.ac.uk/sign-147-management-of-chronic-heart-failure.html

31. Bisoprolol SmPC. 2018 https://www.medicines.org.uk/emc/product/3092/smpc

32. Farmer KC. Methods for measuring and monitoring medication regimen adherence in clinical trials and clinical practice. Clin Therap 1999; 21:1074-1090

33. Skali H, Pfeffer MA, Lubsen J, Solomon SD. Variable impact of combining fatal and nonfatal end points in heart failure trials. Circulation 2006; 114: 2298-2304. 
34. Witek Jr TJ, Mahler DA. Minimal important difference of the transition dyspnoea index in a multinational clinical trial. Eur Respir J 2003; 21: 267-272.

35. The EuroQol Group. EuroQol-a new facility for the measurement of health-related quality of life. Health Policy 1990;16:199-208.

36. COPD Assessment Test. http://www.catestonline.co.uk/

37. Hurst JR, Vestbo J, Anzueto A, Locantore N, Müllerova H, Tal-Singer R, Miller B, Lomas DA, Agusti A, Macnee W, Calverley P, Rennard S, Wouters EF, Wedzicha JA. Evaluation of COPD Longitudinally to Identify Predictive, Surrogate Endpoints. Susceptibility to exacerbation in chronic obstructive pulmonary disease. N Engl J Med 2010;363:1128-1138.

38. Celli BR, MacNee W, ATS/ERS Task Force. Standards for the diagnosis and treatment of patients with COPD: a summary of the ATS/ERS position paper. Eur Resp J 2004;23:932-946.

39. Dolan P, Modelling valuations for health states, Medical Care 1997; 11: 1095-1108.

40. Dodd JW, Hogg L, Nolan J, Jefford H, Grant A, Lord VM, Falzon C, Garrod R, Lee C, Polkey MI, Jones PW, Man WD, Hopkinson NS. The COPD assessment test (CAT): response to pulmonary rehabilitation. A multicentre, prospective study. Thorax 2011;66:425-429.

41. Jones PW, Harding G, Berry P, Wiklund I, Chen WH, Kline Leidy N. Development and first validation of the COPD Assessment Test. Eur Resp J 2009;34:648-654.

42. Mahler DA, Weinberg DH, Wells CK, Feinstein AR. The measurement of dyspnea. Contents, interobserver agreement, and physiologic correlates of two new clinical indexes. Chest 1984; 85: 751758.

43. Graham BL, Steenbruggen I, Barjaktarevic IZ, et al. Standardization of spirometry 2019 update an official American Thoracic Society and European Respiratory Society technical statement. Am J Respir Crit Care Med 2019; 200: E70-88.

44. Barrett B, Byford S. Collecting service use data for economic evaluation in DSPD populations. $\mathrm{Br} \mathrm{J}$ Psychiatry, 2007, 190 (sup 49), s75-s78.

45. Ponikowski P, Voors AA, Anker SD, Bueno H, Cleland JG, Coats AJ, Falk V, Gonzalez-Juanatey JR, Harjola VP, Jankowska EA, Jessup M, Linde C, Nihoyannopoulos P, Parissis JT, Pieske B, Riley JP, Rosano GM, Ruilope LM, Ruschitzka F, Rutten FH, van der Meer P. 2016 ESC Guidelines for the diagnosis and treatment of acute and chronic heart failure. Eur. J Heart Fail.2016;18:891-975.

46. Devereux G, Cotton S, Fielding S, McMeekin N, Barnes PJ, Briggs A, Burns G, Chaudhuri R, Chrystyn H, Davies L, De Soyza A, Gompertz S, Haughney J, Innes K, Kaniewska J, Lee A, Morice A, Norrie J, Sullivan A, Wilson A, Price D. Effect of theophylline as adjunct to inhaled corticosteroids on exacerbations in 
patients with chronic obstructive pulmonary disease: a randomized clinical trial. JAMA 2018; 320: 15481559

47. NICE 2013. Guide to the methods of technology appraisal 2013 https://www.nice.org.uk/process/pmg9/resources/guide-to-the-methods-of-technology-appraisal-2013pdf-2007975843781

48. National Research Ethics Service. Safety and progress reports (CTIMPs). https://www.hra.nhs.uk/approvals-amendments/managing-your-approval/safety-reporting/

49. Alqahtani JS, Oyelade T, Aldhahir AM, Mendes RG, Alghamdi SM, Miravitlles M, Mandal S, Hurst JR. Reduction in hospitalised COPD exacerbations during COVID-19: A systematic review and meta-analysis. PLoS ONE 2021; 16(8): e0255659. https://doi.org/10.1371/journal.pone.0255659

50. Quint JK, Donaldson GC, Hurst JR, Goldring JJP, Seemungal TR, Wedzicha JA. Predictive accuracy of patient-reported exacerbation frequency in COPD. Eur Respir J 2011; 37(3): 501-507.

51. Dransfield MT, Voelker H, Bhatt SP, Brenner K, Casaburi R, Come CE, Cooper JAD, Criner GJ, Curtis JL, Han MLK, Hatipoğlu U, Helgeson ES, Jain VV, Kalhan R, Kaminsky D, Kaner R, Kunisaki KM, Lambert AA, Lammi MR, Lindberg S, Make BJ, Martinez FJ, McEvoy C, Panos RJ, Reed RM, Scanlon PD, Sciurba FC, Smith A, Sriram PS, Stringer WW, Weingarten JA, Wells JM, Westfall E, Lazarus SC, Connett JE.. Metoprolol for the Prevention of Acute Exacerbations of COPD. New Eng J Med 2019;381(24):2304-2314.

52. Baker JG. The selectivity of beta-adrenoceptor antagonists at the human beta1, beta2 and beta3 adrenoceptors. Br J Pharmacol. 2005;144:317-322.

53. Taniguchi A, Miyahara N, Oda N, Morichika D, Ichihara E, Oze I, Tanimoto Y, Ichikawa H, Fujii U, Tanimoto M, Kanehiro A, Kiura K. Protective Effects of Bisoprolol against Acute Exacerbation in Moderate-to-Severe Chronic Obstructive Pulmonary Disease. Acta Med Okayama. $2017 ; 71: 453-457$.

54. Sundh J, Magnuson A, Montgomery S, Andell P, Rindler G, Fröbert O. Beta-blockers to patients with chronic obstructive pulmonary disease (BRONCHIOLE) - Study protocol from a randomized controlled trial. Trials 2020;21:123. doi: 10.1186/s13063-019-3907-1.

\section{Figures}


Recruitment/baseline assessment - assessed for eligibility based on inclusion and exclusion criteria Informed consent taken

Assessment: drug history, smoking history, spirometry ${ }^{1}$, CATest, BDI dyspnoea score, EQ-5D-5L, health care utilisation.

Pulse, blood pressure (BP).

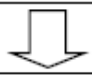

Centralised randomisation $(n=1,574)$

University of Aberdeen, Centre for Healthcare Randomised Trials, web/telephone randomisation service

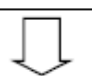

Intervention arm: $\mathrm{n}=787$

Bisoprolol $1.25 \mathrm{mg}$ once daily $(1 \times 1.25 \mathrm{mg}$ tab).

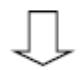

1 week

Pulse, BP, FEV ${ }^{2}$, symptoms, adverse reactions

个Bisoprolol $2.5 \mathrm{mg}$ once daily $(2 \times 1.25 \mathrm{mg} \text { tabs })^{3}$

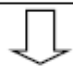

\section{2 weeks}

Pulse, BP, $\mathrm{FEV}_{1}{ }^{2}$, symptoms, adverse reactions

个Bisoprolol $3.75 \mathrm{mg}$ once daily $(3 \times 1.25 \mathrm{mg} \text { tabs })^{3}$

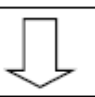

\section{3 weeks}

Pulse, BP, $\mathrm{FEV}_{1}{ }^{2}$, symptoms, adverse reactions $\uparrow$ Bisoprolol $5 \mathrm{mg}$ once daily $(4 \times 1.25 \mathrm{mg} \text { tabs })^{3}$

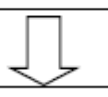

4 weeks

Pulse, BP, $\mathrm{FEV}_{1}{ }^{2}$, symptoms, adverse reactions ${ }^{3}$

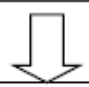

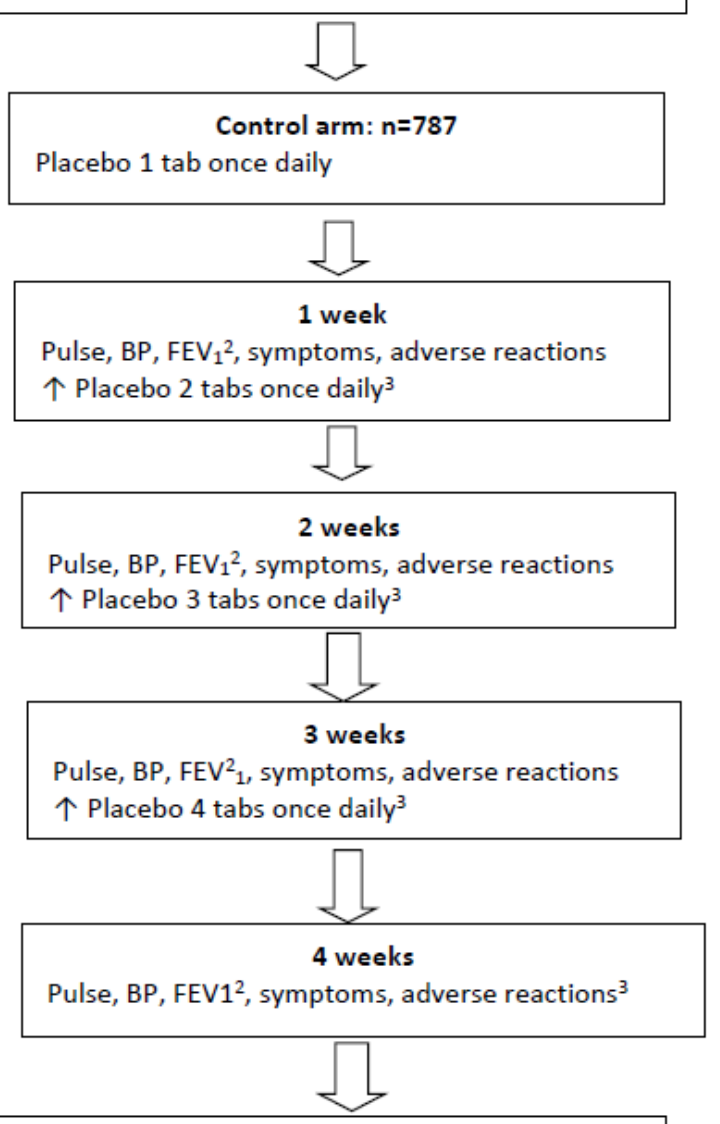

Control arm: $n=787$

1 week

Pulse, $\mathrm{BP}, \mathrm{FEV}_{1}{ }^{2}$, symptoms, adverse reactions

$\uparrow$ Placebo 2 tabs once daily ${ }^{3}$

\section{2 weeks}

Pulse, BP, $\mathrm{FEV}_{1}{ }^{2}$, symptoms, adverse reactions

$\uparrow$ Placebo 3 tabs once daily

\section{3 weeks}

Pulse, BP, $\mathrm{FEV}^{2}{ }_{1}$, symptoms, adverse reactions $\uparrow$ Placebo 4 tabs once daily ${ }^{3}$

\section{4 weeks}

6 month follow-up: exacerbations, hospital admissions, health care utilisation, drug history, spirometry ${ }^{4}$, disease specific health status (CATest), TDI dyspnoea, generic health related quality of life (EQ-5D-5L), adverse reactions, serious adverse events, weight, Pulse, BP.

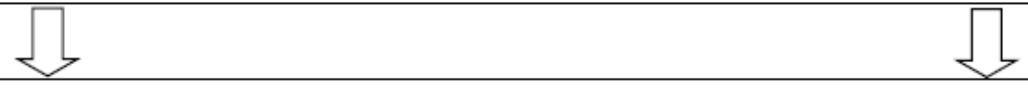

12 month follow-up: exacerbations, hospital admissions, health care utilisation, drug history, spirometry ${ }^{4}$, disease specific health status (CATest), TDI dyspnoea, generic health related quality of life (EQ-5D-5L), adverse reactions, serious adverse events. Pulse, BP. Advise to reduce tablets by one tab a day per week to zero

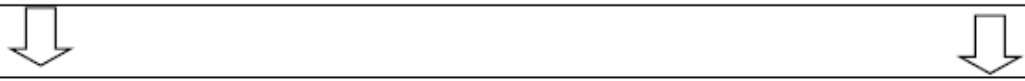

4 week follow-up telephone call: Ensure that medication has been stopped and no adverse events.

\section{Figure 1}

\section{Flow diagram of study design and schedule.}

BDI, baseline dyspnoea index; BP blood pressure; CATest, COPD (Chronic Obstructive Pulmonary Disease) Assessment Test; EQ-5D-5L, EuroQOL five-dimension questionnaire; FEV 1 , forced expiratory volume 1 second; TDI transition dyspnoea index 
${ }^{1}$ During the COVID-19 pandemic, historical spirometry results will be used.

2 During the COVID-19 pandemic, the titration algorithm will consider patient reported breathlessness and not

FEV1.

${ }^{3}$ For further details regarding titration, including cases where there may be down-titration, please see section

8.7 and figure 3.

${ }^{4}$ During the COVID-19 pandemic, 6 and 12 month follow-up will be remote assessments and not include spirometry. 


\begin{tabular}{|c|c|c|c|c|c|c|c|c|}
\hline \multirow[b]{3}{*}{ TIMEPOINT (weeks) } & \multicolumn{8}{|c|}{ STUDY PERIOD } \\
\hline & \multirow{2}{*}{$\begin{array}{c}\text { Enrolment/allocation } \\
0 \\
\end{array}$} & \multicolumn{7}{|c|}{ Post allocation (weeks) } \\
\hline & & 1 & 2 & 3 & 4 & 26 & 52 & 55 \\
\hline \multicolumn{9}{|l|}{ ENROLMENT } \\
\hline \multicolumn{9}{|l|}{ Eligibility screen } \\
\hline Informed consent & $\mathrm{x}$ & & & & & & & \\
\hline \multicolumn{9}{|l|}{ Demographic data } \\
\hline \multicolumn{9}{|l|}{ Clinical history } \\
\hline \multicolumn{9}{|l|}{ Drug history } \\
\hline \multicolumn{9}{|l|}{ Smoking status } \\
\hline \multicolumn{9}{|l|}{ Height } \\
\hline \multicolumn{9}{|l|}{ Weight } \\
\hline \multicolumn{9}{|l|}{ Allocation } \\
\hline Cardiac sub study consent & & & & $x$ & & & & \\
\hline \multicolumn{9}{|l|}{ INTERVENTIONS } \\
\hline \multicolumn{9}{|l|}{ Bisoprolol } \\
\hline \multicolumn{9}{|l|}{ Placebo } \\
\hline \multicolumn{9}{|l|}{ ASSESSMENTS } \\
\hline Pulse/heart rate & $\mathrm{X}$ & $\mathrm{X}$ & $\mathrm{X}$ & $\mathrm{X}$ & $\mathrm{X}$ & $\mathrm{X}$ & $\mathrm{X}$ & \\
\hline Blood pressure & $x$ & $x$ & $x$ & $x$ & $x$ & $x$ & $x$ & \\
\hline $\begin{array}{l}\text { Health related quality of life } \\
\text { EQ-5D-5L }\end{array}$ & $\mathrm{X}$ & & & & & $\mathrm{X}$ & $\mathrm{X}$ & \\
\hline $\begin{array}{l}\text { Disease related health status } \\
\text { (CAT, BDI/TDI dyspnoea) }\end{array}$ & $\mathrm{X}$ & & & & & $\mathrm{X}$ & & \\
\hline $\begin{array}{l}\text { Number COPD exacerbations } \\
\text { requiring OCS/antibiotics }\end{array}$ & & & & & & $x$ & $x$ & \\
\hline Hospital admissions & & & & & & $\mathrm{x}$ & & \\
\hline Adverse events/drug reactions & & $\mathrm{X}$ & $\mathrm{X}$ & $\mathrm{X}$ & $\mathrm{X}$ & $\mathrm{X}$ & $\mathrm{X}$ & \\
\hline Major adverse cardiac events & & & & & & $\mathrm{X}$ & $\mathrm{X}$ & \\
\hline Health care utilisation & $x$ & & & & & $x$ & $x$ & \\
\hline Adherence & & $x$ & $x$ & $x$ & $x$ & $x$ & $\mathrm{X}$ & \\
\hline Echocardiogram (sub-study) & & & & $\mathrm{x}$ & & & & \\
\hline $\begin{array}{l}\text { NT-proBNP, hs-Tnl, Galectin and } \\
\text { hs-CRP (sub study) }\end{array}$ & & & & $x$ & & & $\mathrm{X}$ & \\
\hline $\begin{array}{l}\text { Telephone call to confirm } \\
\text { cessation of study drug }\end{array}$ & & & & & & & & $x$ \\
\hline
\end{tabular}

\section{Figure 2}

Schedule of enrolment, interventions, and assessments.

BDI, baseline dyspnoea index; CATest, COPD (Chronic Obstructive Pulmonary Disease) Assessment Test; EQ-5D-5L, EuroQOL five-dimension questionnaire; hs-cTnl high sensitivity cardiac troponin I; NTproBNP Nterminal pro-Brain Natriuretic PeptideN; OCS oral corticosteroids; TDI transition dyspnoea index 


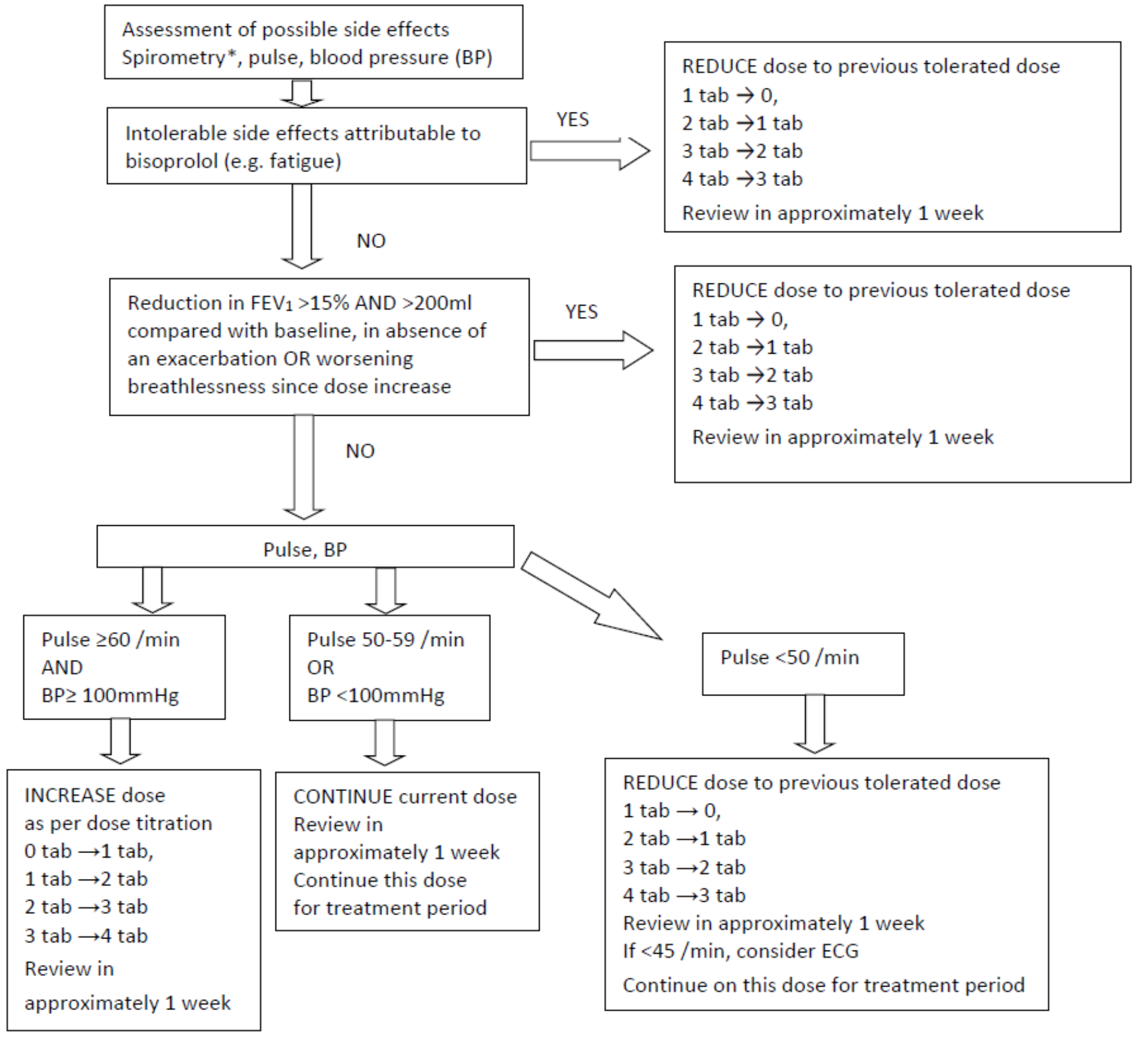

\section{Figure 3}

\section{Schematic representation of dose titration decision making}

*Spirometry not performed during COVID-19, replaced with breathlessness symptoms instead.

\section{Supplementary Files}

This is a list of supplementary files associated with this preprint. Click to download.

- BICSPILversion41Dec2020clean.pdf 
- BICSconsentv330May2018.pdf

- BICSprotocolv714May2021clean.docx 